\title{
Alliance free and alliance cover sets
}

\author{
Juan A. Rodríguez-Velázquez ${ }^{1, *}$, José M. Sigarreta ${ }^{2 \dagger}$ \\ Ismael G. Yero ${ }^{1, \ddagger}$ and Sergio Bermudo ${ }^{3, \S}$ \\ ${ }^{1}$ Department of Computer Engineering and Mathematics \\ Universitat Rovira i Virgili, \\ Av. Països Catalans 26, 43007 Tarragona, Spain. \\ ${ }^{2}$ Faculty of Mathematics \\ Autonomous University of Guerrero \\ Carlos E. Adame 5, Col. La Garita, Acapulco, Guerrero, México \\ ${ }^{3}$ Department of Economy, Quantitative Methods and Economic History \\ Pablo de Olavide University \\ Carretera de Utrera Km. 1, 41013-Sevilla, Spain
}

July 2, 2018

\begin{abstract}
A defensive (offensive) $k$-alliance in $\Gamma=(V, E)$ is a set $S \subseteq V$ such that every $v$ in $S$ (in the boundary of $S$ ) has at least $k$ more neighbors in $S$ than it has in $V \backslash S$. A set $X \subseteq V$ is defensive (offensive) $k$-alliance free, if for all defensive (offensive) $k$-alliance $S, S \backslash X \neq$
\end{abstract}

*e-mail:juanalberto.rodriguez@urv.cat. Partially supported by the Spanish Ministry of Education through projects TSI2007-65406-C03-01 "E-AEGIS" and CONSOLIDER CSD2007-00004 "ARES" and by the Rovira i Virgili University through project 2006AIRE-09

†e-mail:josemaria.sigarreta@uc3m.es

${ }_{\ddagger}^{\ddagger}$ e-mail: ismael.gonzalez@urv.cat

$\S$ e-mail:sbernav@upo.es. Partially supported by Ministerio de Ciencia y Tecnología, ref. BFM2003-00034 and Junta de Andalucía, ref. FQM-260 and ref. P06-FQM-02225. 
$\emptyset$, i.e., $X$ does not contain any defensive (offensive) $k$-alliance as a subset. A set $Y \subseteq V$ is a defensive (offensive) $k$-alliance cover, if for all defensive (offensive) $k$-alliance $S, S \cap Y \neq \emptyset$, i.e., $Y$ contains at least one vertex from each defensive (offensive) $k$-alliance of $\Gamma$. In this paper we show several mathematical properties of defensive (offensive) $k$-alliance free sets and defensive (offensive) $k$-alliance cover sets, including tight bounds on the cardinality of defensive (offensive) $k$-alliance free (cover) sets.

Keywords: Defensive alliance, offensive alliance, alliance free set, alliance cover set.

AMS Subject Classification numbers: 05C69; 05C70

\section{Introduction}

In [2], P. Kristiansen, S. M. Hedetniemi and S. T. Hedetniemi introduced several types of alliances in graphs, including defensive and offensive alliances. We are interested in a generalization of alliances, namely $k$-alliances, given by Shafique and Dutton [4]. In this paper we show several mathematical properties of $k$-alliance free sets and $k$-alliance cover sets.

We begin by stating some notation and terminology. In this paper $\Gamma=$ $(V, E)$ denotes a simple graph of order $n$, size $m$, minimum degree $\delta$ and maximum degree $\Delta$. For a non-empty subset $S \subseteq V$, and any vertex $v \in V$, we denote by $N_{S}(v)$ the set of neighbors $v$ has in $S: N_{S}(v):=\{u \in S: u \sim v\}$ and $\delta_{S}(v)=\left|N_{S}(v)\right|$ denotes the degree of $v$ in $S$. The complement of the set $S$ in $V$ is denoted by $\bar{S}$. The boundary of a set $S \subseteq V$ is defined as $\partial S:=\cup_{v \in S} N_{\bar{S}}(v)$. A nonempty set of vertices $S \subseteq V$ is called a defensive (offensive) $k$-alliance in $\Gamma$ if for every $v \in S(v \in \partial S), \delta_{S}(v) \geq \delta_{\bar{S}}(v)+k$. Hereafter, if there is no restriction on the values of $k$, we assume that $k \in$ $\{-\Delta, \ldots, \Delta\}$. Notice that any vertex subset is an offensive $k$-alliance for $k \in\{-\Delta, 1-\Delta, 2-\Delta\}$.

A set $X \subseteq V$ is defensive (offensive) $k$-alliance free, $k$-daf ( $k$-oaf), if for all defensive (offensive) $k$-alliance $S, S \backslash X \neq \emptyset$, i.e., $X$ does not contain any defensive (offensive) $k$-alliance as a subset $[4,5]$. A defensive (offensive) $k$-alliance free set $X$ is maximal if for every defensive (offensive) $k$-alliance free set $Y, X \not \subset Y$. A maximum $k$-daf ( $k$-oaf) set is a maximal ( $k$-oaf) $k$-daf set of largest cardinality. 
A set $Y \subseteq V$ is a defensive (offensive) $k$-alliance cover, $k$-dac ( $k$-oac), if for all defensive (offensive) $k$-alliances $S, S \cap Y \neq \emptyset$, i.e., $Y$ contains at least one vertex from each defensive (offensive) $k$-alliance of $\Gamma$. A $k$-dac $(k$-oac) set $Y$ is minimal if no proper subset of $Y$ is a defensive (offensive) $k$-alliance cover set. A minimum $k$-dac $(k$-oac) set is a minimal cover set of smallest cardinality.

\section{Remark 1.}

(i) If $X$ is a minimal $k$-dac $(k$-oac) set then, for all $v \in X$, there exists a defensive (offensive) $k$-alliance $S_{v}$ for which $S_{v} \cap X=\{v\}$.

(ii) If $X$ is a maximal $k$-daf ( $k$-oaf) set, then, for all $v \in \bar{X}$, there exists $S_{v} \subseteq X$ such that $S_{v} \cup\{v\}$ is a defensive (offensive) $k$-alliance.

A defensive (offensive) $k$-alliance is global if it is a dominating set. For short, in the case of a global offensive $k$-alliance cover (free) set we will write $k$-goac ( $k$-goaf).

Associated with the characteristic sets defined above we have the following invariants:

$a_{k}(\Gamma)$ : minimum cardinality of a defensive $k$-alliance in $\Gamma$.

$\gamma_{k}(\Gamma)$ : minimum cardinality of a global defensive $k$-alliance in $\Gamma$.

$\gamma_{k}^{o}(\Gamma)$ : minimum cardinality of a global offensive $k$-alliance in $\Gamma$.

$\phi_{k}(\Gamma)$ : cardinality of a maximum $k$-daf set in $\Gamma$.

$\phi_{k}^{o}(\Gamma)$ : cardinality of a maximum $k$-oaf set in $\Gamma$.

$\phi_{k}^{g o}(\Gamma)$ : cardinality of a maximum $k$-goaf set in $\Gamma$.

$\zeta_{k}(\Gamma)$ : cardinality of a minimum $k$-dac set in $\Gamma$.

$\zeta_{k}^{o}(\Gamma)$ : cardinality of a minimum $k$-oac set in $\Gamma$.

$\zeta_{k}^{g o}(\Gamma)$ : cardinality of a minimum $k$-goac set in $\Gamma$.

The following duality between alliance cover and alliance free sets was shown in $[4,5]$.

Remark 2. $X$ is a defensive (offensive) $k$-alliance cover set if and only if $\bar{X}$ is defensive (offensive) $k$-alliance free.

Corollary 3. $\phi_{k}(\Gamma)+\zeta_{k}(\Gamma)=\phi_{k}^{o}(\Gamma)+\zeta_{k}^{o}(\Gamma)=n$. 


\section{Alliance cover and alliance free sets}

We begin by studying the structure of a set according to the structure of its complementary set.

Theorem 4. If $X$ is a minimal $k$-dac set, then $\bar{X}$ is a dominating set.

Proof. By Remark 2 , if $X$ is a minimal $k$-dac set, then $\bar{X}$ is a maximal $k$-daf set. Therefore, for all $v \in X$, there exists $X_{v} \subseteq \bar{X}$ such that $X_{v} \cup\{v\}$ is a defensive $k$-alliance. So, for every $u \in X_{v}, \delta_{X_{v}}(u)+\delta_{\{v\}}(u)=\delta_{X_{v} \cup\{v\}}(u) \geq$ $\delta_{\overline{X_{v} \cup\{v\}}}(u)+k=\delta_{\overline{X_{v}}}(u)-\delta_{\{v\}}(u)+k$. On the other hand, as $X_{v}$ is not a defensive $k$-alliance, there exists $w \in X_{v}$ such that $\delta_{X_{v}}(w)<\delta_{\overline{X_{v}}}(w)+k$. Hence, by the above inequalities, $\delta_{\overline{X_{v}}}(w)+k+\delta_{\{v\}}(w)>\delta_{\overline{X_{v}}}(w)-\delta_{\{v\}}(w)+k$. Thus, $2 \delta_{\{v\}}(w)>0$ and, as a consequence, $v$ is adjacent to $w$.

Notice that there exist minimal $k$-oac sets such that their complement sets are not dominating sets. For instance we consider the graph obtained from the cycle graph $C_{8}$ by adding the edge $\left\{v_{1}, v_{3}\right\}$ and the edge $\left\{v_{5}, v_{7}\right\}$. In this graph the set $S=\left\{v_{2}, v_{3}, v_{5}, v_{6}, v_{7}\right\}$ is a minimal 0 -oac but $\bar{S}$ is not a dominating set.

Theorem 5. If $X$ is a minimal $k$-dac set, then $\bar{X}$ is a global offensive $k$ alliance.

Proof. If $X \subset V$ is a minimal $k$-dac set, then for every $v \in X$ there exists a defensive $k$-alliance $S_{v}$ such that $S_{v} \cap X=\{v\}$. Hence, $\delta_{S_{v}}(v) \geq \delta_{\overline{S_{v}}}(v)+k$ and $\delta_{\bar{X}}(v) \geq \delta_{S_{v}}(v) \geq \delta_{\overline{S_{v}}}(v)+k \geq \delta_{X}(v)+k$. Therefore, for every $v \in X$, we have $\delta_{\bar{X}}(v) \geq \delta_{X}(v)+k$. On the other hand, by Theorem $4, \bar{X}$ is a dominating set. In consequence, $\bar{X}$ is a global offensive $k$-alliance in $\Gamma$.

Corollary 6. $\phi_{k}(\Gamma) \geq \gamma_{k}^{o}(\Gamma)$ and $\zeta_{k}(\Gamma) \leq n-\gamma_{k}^{o}(\Gamma)$.

Notice that if one vertex $v \in V$ belongs to any offensive $k$-alliance, then $V \backslash\{v\}$ is a $k$-oaf set. Hence, $\delta(v)<k$. So, if $k \leq \delta$ and $X$ is a minimal $k$-oac set, then $|X| \geq 2$.

Theorem 7. For every $k \in\{2-\Delta, \ldots, \Delta\}$, if $X$ is a minimal $k$-goac set such that $|X| \geq 2$, then $\bar{X}$ is an offensive $(k-2)$-alliance. Moreover, if $k \in\{3, \ldots, \Delta\}$, then $\bar{X}$ is a global offensive $(k-2)$-alliance. 
Proof. If $X \subset V$ is a minimal $k$-goac set, then for all $v \in X$ there exists a global offensive $k$-alliance, $S_{v}$, such that $S_{v} \cap X=\{v\}$. Hence, $1+\delta_{\bar{X}}(u) \geq$ $\delta_{S_{v}}(u) \geq \delta_{\overline{S_{v}}}(u)+k \geq \delta_{X}(u)+k-1$, for every $u \in \overline{S_{v}}$. As $X \backslash\{v\} \subset \overline{S_{v}}$, we have $\delta_{\bar{X}}(u) \geq \delta_{X}(u)+k-2$ for every $u \in X \backslash\{v\}$. Therefore, $\bar{X}$ is an offensive $(k-2)$-alliance. Moreover, if $k>2, \bar{X}$ is a dominating set. So, in such a case, it is a global offensive $(k-2)$-alliance.

Corollary 8. For every $k \in\{3, \ldots, \delta\}, \phi_{k}^{g o}(\Gamma) \geq \gamma_{k-2}^{o}(\Gamma)$ and $\zeta_{k}^{g o}(\Gamma) \leq n-$ $\gamma_{k-2}^{o}(\Gamma)$.

Theorem 9. For every $k \in\{1-\Delta, \ldots, \Delta-1\}$,

(i) if $X$ is a global offensive $k$-alliance, then $\bar{X}$ is $(1-k)$-daf;

(ii) if $X$ is a defensive $k$-alliance, then $\bar{X}$ is $(1-k)$-goaf.

Proof. (i) If $X$ is a global offensive $k$-alliance, then for every $v \in \bar{X}$ we have $\delta_{X}(v)+1-k>\delta_{\bar{X}}(v)$. Hence, the set $\bar{X}$ is not a defensive $(1-k)$ alliance. Moreover, if $Y \subset \bar{X}$, then for every $y \in Y$ we have $\delta_{\bar{Y}}(y)+1-k \geq$ $\delta_{X}(y)+1-k>\delta_{\bar{X}}(y) \geq \delta_{Y}(y)$. Thus, the set $Y$ is not a defensive $(1-k)$ alliance. Therefore, $\bar{X}$ is a $(1-k)$-daf set.

(ii) If $X$ is a defensive $k$-alliance, then for every $v \in X$ we have $\delta_{\bar{X}}(v)<$ $\delta_{X}(v)+(1-k)$. So, $\bar{X}$ is not a global offensive $(1-k)$-alliance. Moreover, for every $S \subset \bar{X}$ and $v \in X \subset \bar{S}$ it is satisfied $\delta_{S}(v) \leq \delta_{\bar{X}}(v)<\delta_{X}(v)+(1-k) \leq$ $\delta_{\bar{S}}(v)+(1-k)$, in consequence, $S$ is not a global offensive $(1-k)$-alliance.

Corollary 10. For every $k \in\{1-\Delta, \ldots, \Delta-1\}$,

(i) $\zeta_{1-k}(\Gamma) \leq \gamma_{k}^{o}(\Gamma)$ and $\phi_{1-k}(\Gamma) \geq n-\gamma_{k}^{o}(\Gamma)$;

(ii) $\zeta_{1-k}^{g o}(\Gamma) \leq a_{k}(\Gamma)$.

Notice that all equalities in the above corollaries are attained for the complete graph of order $n$ where $\phi_{k}\left(K_{n}\right)=n-\zeta_{k}(\Gamma)=\gamma_{k}^{o}\left(K_{n}\right)=\left\lceil\frac{n+k-1}{2}\right\rceil$ and $\zeta_{1-k}^{g o}(\Gamma)=n-\phi_{1-k}^{g o}(\Gamma)=a_{k}(\Gamma)=\left\lceil\frac{n+k+1}{2}\right\rceil$.

As we show in the following table, by combining some of the above results we can deduce basic properties on alliance free sets and alliance cover sets. For the restrictions on $k$, see the premises of the corresponding results. 


\begin{tabular}{|c|c|}
\hline Rem. 2 and Th. 4 & Any maximal $k$-daf set is a dominating set. \\
\hline Rem. 2 and Th. 5 & Any maximal $k$-daf set is a global offensive $k$-alliance. \\
\hline Rem. 2 and Th. 9 & Any global offensive $k$-alliance is a $(1-k)$-dac set. \\
\hline Th. 5 and Th. 9 & Any minimal $k$-dac set is $(1-k)$-daf. \\
\hline Th. 7 and Th. 9 & Any minimal $k$-goac set of cardinality at least 2 is $(3-k)$-daf. \\
\hline
\end{tabular}

\subsection{Monotony of $\phi_{k}^{g o}(\Gamma)$ and $\phi_{k}(\Gamma)$}

Theorem 11. If $X$ is a $k$-goaf set, $k \in\{1, \ldots, \Delta-2\}$, such that $|X| \leq n-2$, then there exists $v \in \bar{X}$ such that $X \cup\{v\}$ is a $(k+2)$-goaf set.

Proof. Let us suppose that for every $x \in \bar{X}, X \cup\{x\}$ is not a $(k+2)$-goaf set. Let $v \in \bar{X}$ and let $S_{v} \subset X$, such that $S_{v} \cup\{v\}$ is a global offensive $(k+2)$-alliance in $\Gamma$. Then for every $u \in \overline{S_{v} \cup\{v\}}=\overline{S_{v}} \backslash\{v\}$ we have $\delta_{S_{v}}(u)=\delta_{S_{v} \cup\{v\}}(u)-\delta_{\{v\}}(u) \geq \delta_{\overline{S_{v} \cup\{v\}}}(u)-\delta_{\{v\}}(u)+k+2=\delta_{\overline{S_{v}}}(u)-$ $2 \delta_{\{v\}}(u)+k+2 \geq \delta_{\overline{S_{v}}}(u)+k$. So, for every $u \in \bar{X} \backslash\{v\} \subset \overline{S_{v}} \backslash\{v\}, \delta_{X}(u) \geq$ $\delta_{S_{v}}(u) \geq \delta_{\overline{S_{v}}}(u)+k \geq \delta_{\bar{X}}(u)+k$. Now we take a vertex $w \in \bar{X} \backslash\{v\}$ and by the above procedure, taking the vertex $w$ instead of $v$, we obtain that $\delta_{X}(v) \geq \delta_{\bar{X}}(v)+k$. So, $X$ is a global offensive $k$-alliance, a contradiction.

If $X$ is a $k$-goaf for $k \leq \delta$, then $|X| \leq n-2$, as a consequence, the above result can be simplified as follows.

Corollary 12. If $X$ is a $k$-goaf set, $k \in\{1, \ldots, \delta\}$, then there exists $v \in \bar{X}$ such that $X \cup\{v\}$ is a $(k+2)$-goaf set.

It is easy to check the monotony of $\phi_{k}^{g o}$, i.e., $\phi_{k}^{g o}(\Gamma) \leq \phi_{k+1}^{g o}(\Gamma)$. As we can see below, Theorem 11 leads to an interesting property about the monotony of $\phi_{k}^{g o}$.

Corollary 13. For every $k \in\{1, \ldots, \min \{\delta, \Delta-2\}\}$ and $r \in\left\{1, \ldots,\left\lfloor\frac{\Delta-k}{2}\right\rfloor\right\}$, $\phi_{k}^{g o}(\Gamma)+r \leq \phi_{k+2 r}^{g o}(\Gamma)$.

Theorem 14. If $X$ is a $k$-daf set and $v \in \bar{X}$, then $X \cup\{v\}$ is $(k+2)-d a f$.

Proof. Let us suppose that there exists a defensive $(k+2)$-alliance $A$ such that $A \subseteq X \cup\{v\}$. If $v \notin A$, then $A \subset X$, a contradiction because every defensive (k+2)-alliance is a defensive $k$-alliance. If $v \in A$, let $B=A \backslash\{v\}$. As for every $u \in B, \delta_{B}(u)=\delta_{A}(u)-\delta_{\{v\}}(u)$ and $\delta_{\bar{B}}(u)=\delta_{\bar{A}}(u)+\delta_{\{v\}}(u)$, we have, $\delta_{A}(u) \geq \delta_{\bar{A}}(u)+k+2 \delta_{B}(u)+\delta_{\{v\}}(u) \geq \delta_{\bar{B}}(u)-\delta_{\{v\}}(u)+k+2 \delta_{B}(u) \geq \delta_{\bar{B}}(u)+k$. So, $B \subseteq X$ is a defensive $k$-alliance, a contradiction. 
Corollary 15. For every $k \in\{-\Delta, \ldots, \Delta-2\}$ and $r \in\left\{1, \ldots,\left\lfloor\frac{\Delta-k}{2}\right\rfloor\right\}, \phi_{k}(\Gamma)+$ $r \leq \phi_{k+2 r}(\Gamma)$.

\section{Tight bounds}

A dominating set $S \subset V$ is a global boundary offensive $k$-alliance if for every $v \in \bar{S}, \delta_{S}(v)=\delta_{\bar{S}}(v)+k[6]$.

Lemma 16. If $\{X, Y\}$ is a vertex partition of a graph $\Gamma$ into two global boundary offensive 0-alliances, then $X$ and $Y$ are minimal global offensive 0 -alliances in $\Gamma$.

Proof. Let us suppose, for instance, that $X$ is not a minimal global offensive 0 -alliances, then, there exists $A \subset X$, such that, $X \backslash A \neq \emptyset$ and $A$ is a global offensive 0 -alliance. Thus, for every $v \in \bar{A}, \delta_{X}(v) \geq \delta_{A}(v) \geq \delta_{\bar{A}}(v) \geq \delta_{Y}(v)$.

As $Y \subset \bar{A}$ and $\{X, Y\}$ is a vertex partition of the graph into two global boundary offensive 0 -alliances, then for every $v \in Y, \delta_{Y}(v)=\delta_{X}(v) \geq$ $\delta_{A}(v) \geq \delta_{\bar{A}}(v) \geq \delta_{Y}(v)$.

Therefore, as $Y$ is a dominating set, the above expression carry out just in the case that $A=X$, a contradiction. So, $X$ and $Y$ are minimal global offensive 0 -alliances.

Theorem 17. For every $k \in\{0, \ldots, \Delta\}, \phi_{k}^{g o}(\Gamma) \geq\left\lfloor\frac{n}{2}\right\rfloor+\left\lfloor\frac{k}{2}\right\rfloor-1$.

Proof. First, we will prove the case $k=0$. Let $\{X, Y\}$ be a partition of the vertex set, such that $|X|=\left\lfloor\frac{n}{2}\right\rfloor,|Y|=\left\lceil\frac{n}{2}\right\rceil$ and there is a minimum number of edges between $X$ and $Y$. If $X$ (or $Y$ ) is a 0 -goaf set, then $\phi_{0}^{g o}(\Gamma) \geq\left\lfloor\frac{n}{2}\right\rfloor-1$. We suppose there exist $A \subset X$ and $B \subset Y$, such that $A$ and $B$ are global offensive 0-alliances. Hence $\delta_{X}(v) \geq \delta_{A}(v) \geq \delta_{\bar{A}}(v) \geq \delta_{Y}(v), \quad \forall v \in \bar{A}$, and $\delta_{Y}(v) \geq \delta_{B}(v) \geq \delta_{\bar{B}}(v) \geq \delta_{X}(v), \quad \forall v \in \bar{B}$. As $Y \subset \bar{A}$ and $X \subset \bar{B}$ we have, for every $v \in Y, \delta_{X}(v) \geq \delta_{Y}(v)$ and for every $v \in X, \delta_{Y}(v) \geq \delta_{X}(v)$.

For any $y \in Y$ and $x \in X$, let us take $X^{\prime}=X \backslash\{x\} \cup\{y\}$ and $Y^{\prime}=Y \backslash\{y\} \cup\{x\}$. If $\delta_{X}(y)>\delta_{Y}(y)$ or $\delta_{Y}(x)>\delta_{X}(x)$ then, the edge cutset between $X^{\prime}$ and $Y^{\prime}$ is lesser than the other one between $X$ and $Y$, a contradiction. Therefore $\delta_{X}(y)=\delta_{Y}(y)$ and $\delta_{Y}(x)=\delta_{X}(x)$ and, as a consequence, $\{X, Y\}$ is a partition of the vertex set into two global boundary offensive 0 -alliances. Now, by using Lemma 16 we obtain that $X$ and $Y$ are minimal global offensive 0 -alliances. As a consequence, $\phi_{0}^{g o}(\Gamma) \geq\left\lfloor\frac{n}{2}\right\rfloor-1$. 
Now, let us prove the case $k>0$. Case $1: \phi_{k}^{g o}(\Gamma) \geq n-2$. Since $n-1 \geq\left\lfloor\frac{2 n-1}{2}\right\rfloor \geq\left\lfloor\frac{n+\Delta}{2}\right\rfloor \geq\left\lfloor\frac{n+k}{2}\right\rfloor \geq\left\lfloor\frac{n}{2}\right\rfloor+\left\lfloor\frac{k}{2}\right\rfloor$, we have $\phi_{k}^{g o}(\Gamma) \geq\left\lfloor\frac{n}{2}\right\rfloor+\left\lfloor\frac{k}{2}\right\rfloor-1$. Case 2: $\phi_{k}^{g o}(\Gamma)<n-2$. As every $k$-goaf set is also a $(k+1)$-goaf set, $\phi_{1}^{g o}(\Gamma) \geq \phi_{0}^{g o}(\Gamma) \geq\left\lfloor\frac{n}{2}\right\rfloor+\left\lfloor\frac{1}{2}\right\rfloor-1$, then the statement is true for $k=1$. Hence, we will proceed by induction on $k$. Let us assume that the statement is true for an arbitrary $k \in\{2, \ldots, \Delta-2\}$, that is, there exists a maximal $k$-goaf set $X$ in $\Gamma$ such that, $|X|=\phi_{k}^{g o}(\Gamma) \geq\left\lfloor\frac{n}{2}\right\rfloor+\left\lfloor\frac{k}{2}\right\rfloor-1$. Now, by Theorem 11 , there exists $v \in \bar{X}$, such that $X \cup\{v\}$ is a $(k+2)$-goaf set. Therefore, $\phi_{k+2}^{g o}(\Gamma) \geq|X \cup\{v\}| \geq\left\lfloor\frac{n}{2}\right\rfloor+\left\lfloor\frac{k}{2}\right\rfloor=\left\lfloor\frac{n}{2}\right\rfloor+\left\lfloor\frac{k+2}{2}\right\rfloor-1$. So, the proof is complete.

The above bound is attained, for instance, in the case of the complete graph if $n$ and $k$ are both even or if $n$ and $k$ have different parity: $\phi_{k}^{g o}\left(K_{n}\right)=$ $\left\lfloor\frac{n+k-2}{2}\right\rfloor$.

Theorem 18. $\left\lceil\frac{\delta+k-2}{2}\right\rceil \leq \phi_{k}^{o}(\Gamma) \leq\left\lfloor\frac{2 n-\delta+k-3}{2}\right\rfloor$.

Proof. If $X$ is a $k$-oaf set, then $\delta_{X}(v)+1 \leq \delta_{\bar{X}}(v)+k$, for some $v \in \partial X$. Therefore, $\delta(v)+1-k=\delta_{X}(v)+\delta_{\bar{X}}(v)+1-k \leq 2 \delta_{\bar{X}}(v) \leq 2(n-|X|-1)$. Thus, the upper bound is deduced.

If $X$ is a maximal $k$-oaf set, then $\bar{X}$ is a minimal $k$-oac set. Thus, for all $v \in \bar{X}$, there exists an offensive $k$-alliance $S_{v}$ such that $S_{v} \cap \bar{X}=\{v\}$. Hence, $\delta_{S_{v}}(u) \geq \delta_{\overline{S_{v}}}(u)+k$, for every $u \in \partial S_{v}$. Therefore, $\delta(u)+k \leq 2 \delta_{S_{v}}(u) \leq$ $2\left|S_{v}\right| \leq 2(|X|+1)$. Thus, the lower bound follows.

The above bounds are attained, for instance, for the complete graph: $\phi_{k}^{o}\left(K_{n}\right)=\left\lceil\frac{n+k-3}{2}\right\rceil$.

For every $k \in\{0, \ldots, \Delta\}$ it was established in [5] that $\phi_{k}(\Gamma) \geq\left\lfloor\frac{n}{2}\right\rfloor+\left\lfloor\frac{k}{2}\right\rfloor$. The next result shows other bounds on $\phi_{k}(\Gamma)$.

Theorem 19. For any connected graph $\Gamma,\left\lceil\frac{n(k+\mu)-\mu}{n+\mu}\right\rceil \leq \phi_{k}(\Gamma) \leq\left\lfloor\frac{2 n+k-\delta-1}{2}\right\rfloor$, where $\mu$ denotes the algebraic connectivity of $\Gamma$.

Proof. It was shown in [3] that the defensive $k$-alliance number is bounded by $a_{k}(\Gamma) \geq\left[\frac{n(\mu+k+1)}{n+\mu}\right]$. On the other hand, if $S$ is a defensive $k$-alliance of cardinality $a_{k}(\Gamma)$, then for all $v \in S$ we have that $S \backslash\{v\}$ is a $k$-daf set. Thus, $\phi_{k}(\Gamma) \geq a_{k}(\Gamma)-1$. Hence, the lower bound on $\phi_{k}(\Gamma)$ follows.

Moreover, if $X$ is a $k$-daf set, then $\delta_{X}(v)+1 \leq \delta_{\bar{X}}(v)+k, \quad$ for some $\quad v \in$ $X$. Therefore, $\delta(v)+1-k=\delta_{X}(v)+\delta_{\bar{X}}(v)+1-k \leq 2 \delta_{\bar{X}}(v) \leq 2(n-|X|)$. Thus, the upper bound follows. 
The above bound is sharp as we can check, for instance, for the complete graph $\Gamma=K_{n}$. As the algebraic connectivity of $K_{n}$ is $\mu=n$, the above theorem gives the exact value of $\phi_{k}\left(K_{n}\right)=\left\lceil\frac{n+k-1}{2}\right\rceil$.

Theorem 20. For any connected graph $\Gamma, \zeta_{k}(\Gamma) \leq \frac{n}{\mu_{*}}\left(\mu_{*}-\left\lceil\frac{\delta+k}{2}\right\rceil\right)$, where $\mu_{*}$ denotes the Laplacian spectral radius of $\Gamma$.

Proof. The result immediately follows from Corollary 6 and the following bound obtained in [1]: $\gamma_{k}^{o}(\Gamma) \geq \frac{n}{\mu_{*}}\left\lceil\frac{\delta+k}{2}\right\rceil$.

The above bound is tight as we can check, for instance, for the complete graph $\Gamma=K_{n}$. As the Laplacian spectral radius of $K_{n}$ is $\mu_{*}=n$, the above theorem gives the exact value of $\zeta_{k}\left(K_{n}\right)=\left\lceil\frac{n-k}{2}\right\rceil$.

\section{References}

[1] H. Fernau, J.A. Rodríguez and J.M. Sigarreta, Offensive k-alliances in graphs. Discrete Applied Mathematics 157 (2) (2009), 177-182.

[2] S. M. Hedetniemi, S. T. Hedetniemi, and P. Kristiansen, Alliances in graphs. J. Combin. Math. Combin. Comput. 48 (2004), 157-177.

[3] J.A. Rodríguez, I. G. Yero and J.M. Sigarreta, Defensive $k$-alliances in graphs. Applied Mathematics Letter 22 (2009), 96-100.

[4] K. H. Shafique and R. D. Dutton, Maximum alliance-free and minimum alliance-cover sets, Congr. Numer. 162 (2003),139-146.

[5] K. H. Shafique y R. Dutton, A tight bound on the cardinalities of maximun alliance-free and minimun alliance-cover sets, J. Combin. Math. Combin. Comput. 56 (2006) 139-145.

[6] I. G. Yero and J. A. Rodríguez-Velázquez, Boundary offensive $k$-alliances in graphs. Submitted, 2008. 